\title{
IMPLANTE AUDITIVO DE TRONCO ENCEFÁLICO: REVISÃO DE LITERATURA
}

\section{Auditory brainstem implant: a review}

\author{
Sinéia Neujahr dos Santos ${ }^{(1)}$, Tania Maria Tochetto ${ }^{(2)}$
}

\begin{abstract}
RESUMO
Objetivo: pesquisar aspectos relevantes sobre implante auditivo de tronco encefálico como conceito, finalidade, métodos de implantação, indicações do procedimento e benefícios. Métodos: realizou-se compilação dos trabalhos disponíveis nas bases de dados LILACS, MEDLINE e SCIELO utilizando os descritores: implante auditivo de tronco encefálico, reabilitação de deficientes auditivos, perda auditiva neurossensorial, procedimentos cirúrgicos otológicos. Resultados: o implante auditivo de tronco encefálico é semelhante ao implante coclear, exceto pela configuração do eletrodo que é projetado para ser colocado no complexo do núcleo coclear, no tronco encefálico. Originalmente foi desenvolvido para restaurar a audição em pacientes com ausência ou lesão no nervo coclear. Hoje é indicado para pacientes que por motivos anatômicos ou funcionais não podem receber estímulos elétricos pela orelha interna, como em casos de malformação/ausência de nervo coclear, ossificação coclear e neuropatia auditiva. Os efeitos relatados são: restabelecimento da audição em pacientes com perda auditiva total, níveis de detecção e discriminação dos sons similares às obtidas com implante coclear e acesso a sons ambientais e da fala. Conclusão: o implante auditivo de tronco encefálico proporciona o restabelecimento da sensação auditiva em sujeitos não beneficiados pelo uso de próteses auditivas e implante coclear. A quase totalidade dos pacientes implantados obteve algum benefício na comunicação.
\end{abstract}

DESCRITORES: Implante Auditivo de Tronco Encefálico; Reabilitação de Deficientes Auditivos; Perda Auditiva Neurossensorial; Procedimentos Cirúrgicos Otológicos

\section{INTRODUÇÃO}

A perda auditiva de grau profundo tem conseqüências devastadoras para o desenvolvimento e a manutenção da comunicação. A incapacidade de ouvir os sons do ambiente afeta a qualidade de vida dos adultos ${ }^{1}$ e é extremamente preocupante para o desenvolvimento da linguagem e aprendizagem em crianças ${ }^{2}$.

Quando o uso de aparelhos auditivos não proporciona benefícios o implante coclear tem sido indicado ${ }^{3}$. Porém, dependendo da localização da lesão, este tipo de intervenção nem sempre traz resultados satisfatórios ${ }^{4}$.

(1) Fonoaudióloga do Projeto Promoção da Saúde Auditiva da Universidade Federal de Santa Maria; Mestranda em Distúrbios da Comunicação Humana pela Universidade Federal de Santa Maria.

(2) Fonoaudióloga; Professora Adjunta do Departamento de Fonoaudiologia da Universidade Federal de Santa Maria; Doutora em Ciências dos Distúrbios da Comunicação Humana pela Universidade Federal de São Paulo.
O implante auditivo de tronco encefálico foi desenvolvido para restaurar a audição em pacientes que apresentam ausência ou lesão no nervo coclear ${ }^{5}$.

O objetivo deste estudo foi pesquisar aspectos relevantes sobre implante auditivo de tronco encefálico, tais como conceito, finalidade, métodos de implantação, benefícios e indicações do procedimento.

\section{MÉTODOS}

Foi realizado um levantamento bibliográfico sobre o assunto nas bases de dados eletrônicas LILACS, MEDLINE e SCIELO. Utilizou-se a combinação de palavras-chaves relacionadas ao tema: auditory brainstem implantation e auditory rehabilitation. Foram selecionados estudos realizados desde a década de 90 até os dias atuais. A análise dos dados foi realizada conforme a relevância e o valor informativo de tais materiais para os objetivos do estudo. 


\section{RESULTADOS}

O implante auditivo de tronco encefálico (Auditory Brainstem Implant - ABI) foi desenvolvido para restaurar alguma audição útil em pacientes que apresentam ausência de nervo coclear bilateralmente ${ }^{5}$.

O que diferencia o implante auditivo de tronco encefálico e o implante coclear é a configuração do eletrodo, projetado para ser introduzido ao nível do nervo coclear e não na rampa timpânica da cóclea.

Beneficiam-se do implante auditivo de tronco encefálico pacientes que por motivos anatômicos ou funcionais não possam receber estímulos elétricos pela orelha interna ${ }^{6}$.

O implante auditivo de tronco encefálico foi desenvolvido primeiramente com um eletrodo monocanal no House Ear Institute, em Los Angeles, Califórnia. Este primeiro modelo foi utilizado entre 1979 e 1992 com resultados clínicos precários. A partir desta experiência foi desenvolvido o implante multicanal, em uma parceria do House Ear Institute, da Cochlear Corporation (Englewood, Colorado) e a Huntington Medical Research Institute (Pasadena, Califórnia).

Apesar de a primeira cirurgia para implantação de eletrodo auditivo em tronco encefálico datar de 1979, apenas em outubro de 2000 houve aprovação para seu uso clínico ${ }^{7}$.

Até 2005, nem um único paciente havia recebido este tipo de dispositivo no Brasil 8 .
O eletrodo do implante auditivo de tronco encefálico é introduzido na parte lateral baixa do quarto ventrículo e colocado sobre a superfície dos núcleos cocleares ventral e dorsal (Figura 1). O estímulo elétrico emitido pelo implante auditivo de tronco encefálico possibilita a identificação da freqüência, amplitude e características temporais dos sons ${ }^{9}$.

A técnica cirúrgica utilizada para a implantação do eletrodo no tronco encefálico é semelhante à utilizada na remoção de neurinoma vestibular. O eletrodo é colocado no complexo do núcleo coclear, composto pelos núcleos coclear ventral e dorsal. O núcleo coclear ventral é o principal núcleo de transmissão de impulsos neurais do VIII par. Seus axônios formam a principal via ascendente do nervo coclear $^{8}$.

Podem ser adotadas duas abordagens cirúrgicas: através do labirinto (translabiríntica) ou abaixo do lobo occipital (suboccipital) ${ }^{10}$.

Tanto o núcleo ventral como o dorsal não são visíveis durante a cirurgia e sua localização depende de identificação de estruturas anatômicas adjacentes ${ }^{8}$. A via de acesso escolhida deve ser ampla o suficiente para permitir a correta identificação dos parâmetros anatômicos utilizados como referência ao correto posicionamento dos eletrodos. A opção depende da experiência do cirurgião com cada um deles. A maioria dos otorrinolaringologistas opta pelo acesso translabiríntico em cirurgias para a retirada de neurinomas vestibulares grandes ou com audição deteriorada. Portanto,

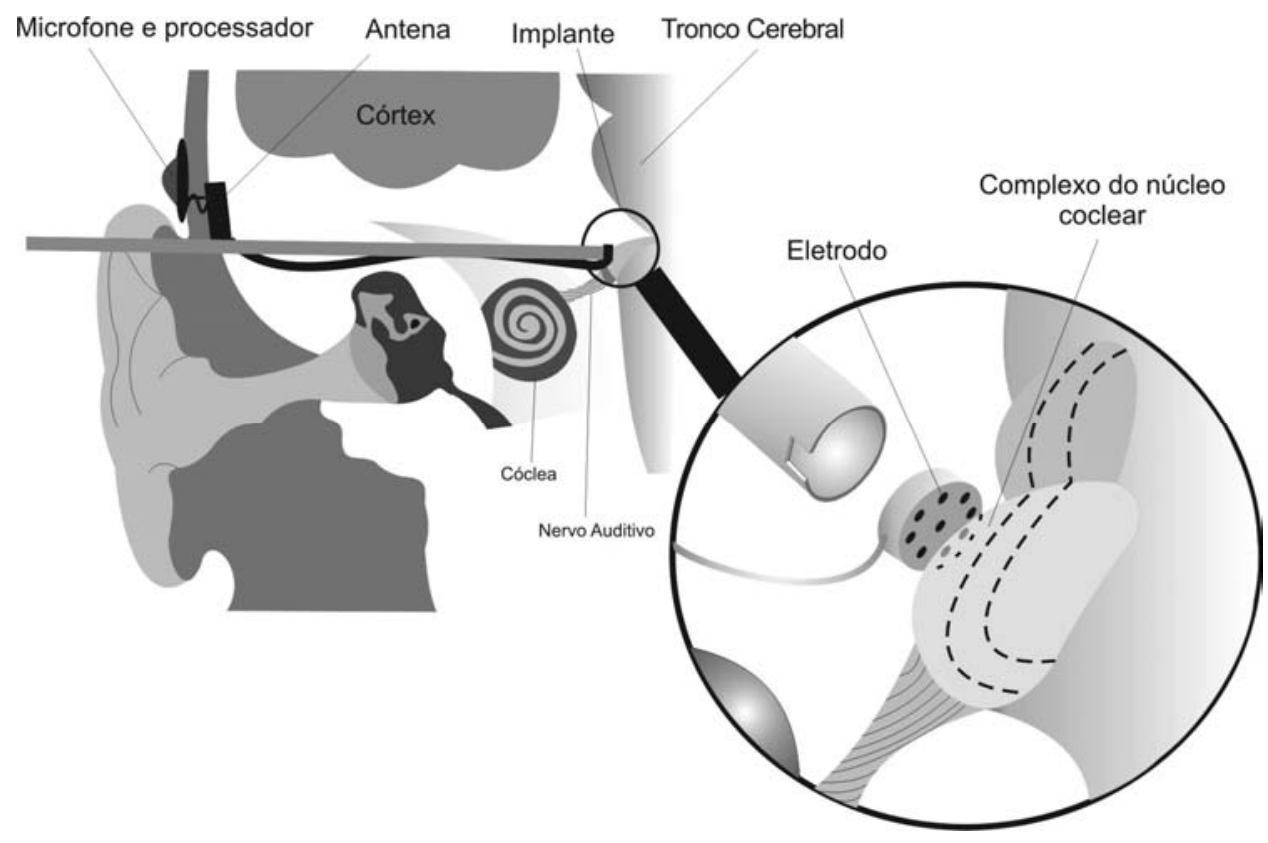

Fonte: www.newscientist.com/channel/being-human/brain/dn4540 (com termos traduzidos para o Português).

Figura 1 - Local onde o eletrodo é introduzido: sobre a superfície dos núcleos cocleares ventral e dorsal 


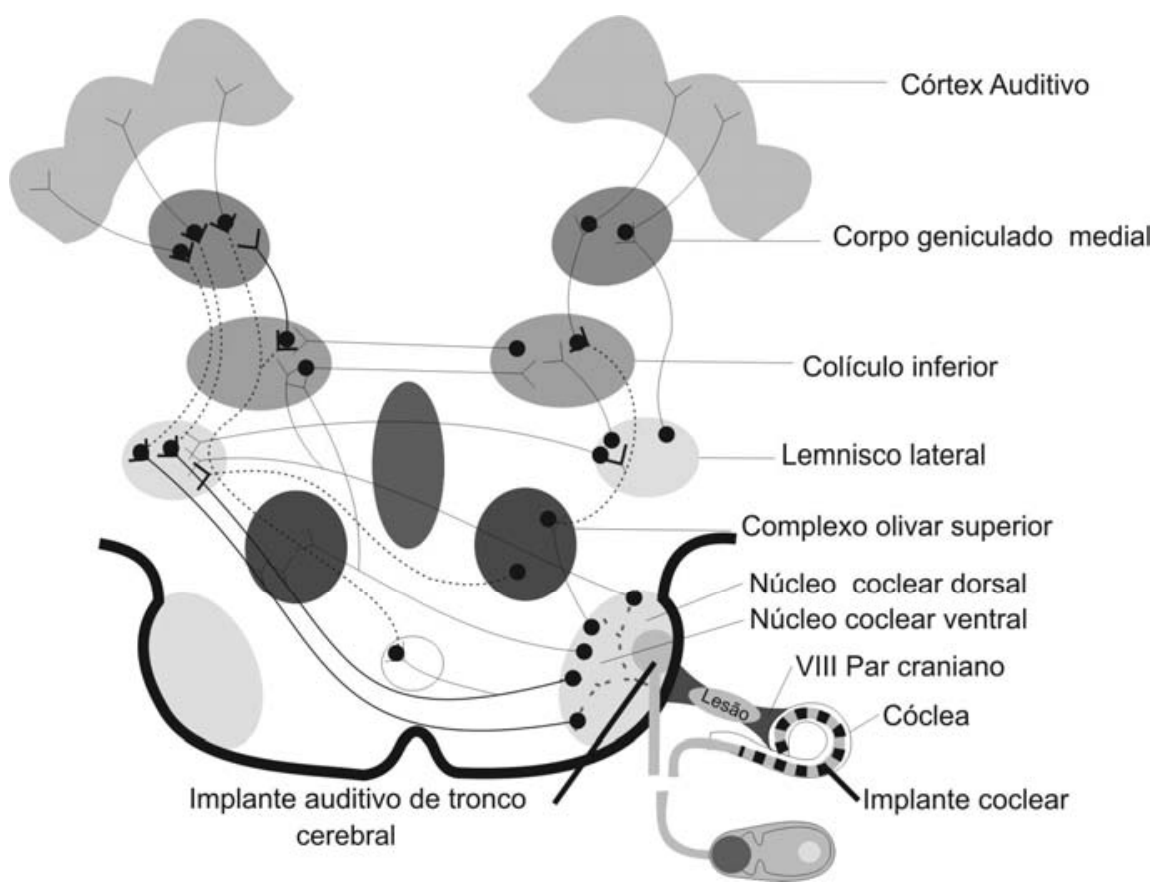

Fonte: www.neurinoma.com.br/abi.html (com termos traduzidos para o Português).

Figura 2 - Vias de estimulação

realizando a implantação no mesmo tempo cirúrgico, este acesso é o escolhido ${ }^{11}$.

O acesso suboccipital é tradicionalmente o preferido dos neurocirurgiões. É bastante seguro e permite a exposição ampla da fossa cerebral posterior mostrando as relações do tumor com os pares cranianos bulbares e grandes vasos. Contudo requer retração cerebelar extensa, o que causa desequilíbrio no pós-operatório, além de não permitir a identificação precoce do nervo facial ${ }^{11}$.

Assim como no implante coclear, um processador é anexado ao corpo do paciente e uma bobina ou um plugue da transmissão, colocada na pele, transmitem o som ao implante. O microfone capta o som e o transmite até o processador do sinal. O processador seleciona as características particulares do som e as codifica eletronicamente. O sinal então é enviado a um transmissor, que emite o sinal ao receptor. O sinal elétrico então é enviado ao longo de um fio até os núcleos cocleares do tronco encefálico (responsáveis pela audição). A partir deste momento o impulso continua seu caminho, até as áreas auditivas do córtex cerebral ${ }^{12}$ (Figura 2).

O implante auditivo de tronco encefálico realmente restabelece a audição em pacientes com perda auditiva total. Ele proporciona níveis de detecção e discriminação dos sons similares às fornecidas pelo implante coclear, mas sem organização tonotópica, pois ele estimula regiões do nervo conforme o número de eletrodos ativados ${ }^{13}$.
A programação de um implante auditivo de tronco encefálico é semelhante à programação de um implante coclear, com a exceção de que alguns pacientes talvez não percebam diferenças em pitch e um número maior de pacientes experimentem sensações não-auditivas ${ }^{14}$.

A ativação do implante é feita aproximadamente cinco semanas após a cirurgia, em unidade de terapia intensiva, com monitoramento em função dos riscos envolvidos. Os eletrodos são ativados e estimulados até que o paciente perceba alguma modificação auditiva. São freqüentes efeitos colaterais da ativação como tontura, desconforto/dormência na laringe, nos braços e nas pernas.

Em adultos a estimulação permite reconhecimento de sentenças com e sem apoio visual aproximadamente em 6-9 meses após a ativação e alguns conseguem utilizar o telefone em conversas simples. Crianças respondem os testes instrumentais e reconhecem a voz após aproximadamente três meses da ativação.

A inteligibilidade da fala propiciada pelo implante auditivo de tronco encefálico costuma ser mais clara se associada à leitura labial. $O$ implante auditivo de tronco encefálico mostrou-se eficaz na compreensão de frases, mas os acertos são 35\% em média melhores se utilizada a leitura labial conjuntamente, podendo chegar a ter uma melhora de até $75 \%{ }^{3}$.

O implante auditivo de tronco encefálico permite o acesso a sons ambientais e da fala, mas 
poucos pacientes conseguem compreender a fala apenas com a audição. Usando o implante auditivo de tronco encefálico para distinguir sons e compreender da fala, a maioria dos pacientes consegue atingir uma média de $65 \%$ em testes de reconhecimento de frases, o que representa um aumento em cerca de $30 \%$ comparado à compreensão de fala sem utilização de apoio visual ${ }^{14}$.

Antes do procedimento o paciente deve estar ciente das várias etapas a serem percorridas até o uso pleno do implante auditivo de tronco encefálico: os riscos do processo cirúrgico; a ativação gradual dos eletrodos no período pós-operatório e seus riscos. A reabilitação auditiva é lenta, em decorrência da ativação gradativa do implante auditivo de tronco encefálico e limitada, pois a audição não será restabelecida por completo e a identificação tonotópica dos sons, inicialmente, não será possível. Os sujeitos implantados devem ter disponibilidade para o acompanhamento intensivo requerido no primeiro ano após a cirurgia.

Candidatos em potencial ao implante auditivo de tronco encefálico precisam estar dispostos a aceitar as limitações do dispositivo e estarem altamente motivados a utilizá-lo no seu dia-a-dia. Isso é essencial para prolongar o desempenho e os benefícios do implante auditivo de tronco encefálico ${ }^{14}$.

\section{Indicações do Implante Auditivo de Tronco Encefálico}

Os pacientes que classicamente se beneficiariam deste tipo de prótese auditiva eletrônica cirurgicamente implantável são aqueles com diagnóstico de neurofibromatose tipo 2 (NF-2), por apresentarem neurinomas vestibulares bilaterais ou crianças com aplasia congênita de nervo coclear. Em pacientes que perderam a audição após a completa remoção do tumor, o implante auditivo de tronco encefálico pode ser uma opção para permitir a continuidade das sensações auditivas.

Atualmente a indicação do implante auditivo de tronco encefálico se ampliou e pacientes com integridade neural do VIII par e impossibilidade de colocação de um implante coclear convencional, como portadores de cócleas ossificadas após meningite ${ }_{8,15,16}$, raros casos de neuropatia central ${ }^{8}$, neuropatia auditiva severa, ausência do nervo auditivo e malformação coclear severa ${ }^{15}$.

Em países economicamente avançados, a principal causa da perda estrutural das vias auditivas periféricas bilateralmente é a neurofibromatose tipo 2, cuja característica essencial é a de evoluir com neurinomas vestibulares bilaterais. Em pacientes com tumores de grandes dimensões com compressão do tronco encefálico, mesmo quando há boa audição, o procedimento mais comum é a completa remoção cirúrgica sem preservação da audição ${ }^{6}$. Porém, isto não ocorre no Brasil, onde as etiologias infecciosas ainda são responsáveis pela maioria dos casos de surdez, dentre elas a meningite. Nestes casos o implante coclear multicanal é o recurso mais utilizado. Este fato é preocupante, uma vez que o prognóstico da função auditiva após a implantação está intimamente relacionado à quantidade de elementos neurais viáveis e ao correto posicionamento dos eletrodos na cóclea. A meningite contraria estes dois fatores: é a etiologia que mais destrói células ciliadas da cóclea e neurônios do nervo coclear e comumente causa ossificação da cápsula ótica ${ }^{8}$.

\section{Implante Auditivo de Tronco Encefálico em casos de neurinoma}

A cirurgia para retirada no neurinoma associada à colocação do implante auditivo de tronco encefálico foi relatada por vários estudos ${ }^{6,12,14,17}$. Nesta pesquisa em $2000{ }^{6}$ foram relatados os resultados obtidos em oito pacientes que receberam o implante auditivo de tronco encefálico, três deles por ocasião da cirurgia de remoção do neurinoma. Em seis casos foram detectadas respostas perceptivas e psicofísicas aos sons após a cirurgia. Em um sujeito a colocação do eletrodo não obteve sucesso e apenas sensações não auditivas foram referidas. Cinco pacientes passaram a utilizar o implante regularmente. $O$ correto posicionamento dos eletrodos foi possível em sete pacientes, o que foi confirmado pela avaliação dos Potenciais Auditivos Evocados de Troco Encefálico em quatro deles.

Em estudo em $2002{ }^{12}$, a ativação dos eletrodos proporcionou sensações auditivas na maioria dos pacientes $(n=25)$, com resultados semelhantes ao do implante coclear monocanal.

Em outro estudo de $2002{ }^{14}$, implantaram dispositivo com oito eletrodos, que foram ativados aproximadamente seis meses após a cirurgia. Os 61 pacientes foram avaliados com uma bateria de testes psicofísicos e de percepção de fala, a cada três meses no primeiro ano, depois da estimulação inicial e depois anualmente. Os autores concluíram que o implante auditivo de tronco encefálico promoveu o restabelecimento das habilidades comunicativas, tanto de detecção quanto de compreensão dos sons.

Em pesquisa realizada em $2005^{17}$, foi realizado o implante auditivo de tronco encefálico em 10 sujeitos ( 9 adultos e 1 criança) com neurinoma bilateralmente e três adultos com neurinoma unilateral. Todos os pacientes relataram sensações auditivas. Os resultados percentuais nos testes de detecção e compreensão de sons, palavras e frases diferiram 
entre os sujeitos. Realizou-se acompanhamento regularmente no primeiro e sexto mês e ao final de um ano após a ativação dos eletrodos. Após este período houve retornos para reavaliação.

\section{Implante Auditivo de Tronco Encefálico em casos de malformações da cóclea e ausência de nervo coclear}

Pesquisadores ${ }^{3,17-20}$ relataram seus resultados obtidos em adultos e crianças com malformações cocleares e aplasia do nervo coclear. As habilidades comunicativas melhoraram para vários dos sujeitos implantados.

Adultos e crianças com 2 a 4 anos de idade também foram implantados ${ }^{18,19}$.

A ativação dos eletrodos foi feita 30 dias após a implantação, 30 a 60 dias ${ }^{19}$ e de dois a 16 meses ${ }^{3}$.

O tempo decorrido entre a ativação dos eletrodos e o início dos progressos na comunicação variou de um a 15 meses ${ }^{17-19}$.

Os benefícios verificados na comunicação após a implantação do implante auditivo de tronco encefálico e a ativação dos eletrodos foram: consciência dos sons ambientais ${ }^{18,19}$; moderada discriminação de fala e boa detecção associada a pouca discriminação da fala ${ }^{18}$; detecção de fala que variou de boa a moderada, e razoável compreensão ${ }^{19}$; acertos entre 40 e $100 \%$ no reconhecimento de palavras e de 60 e $100 \%$ no reconhecimento de sentenças e habilidade de acompanhar a conversação ${ }^{3}$.

Implante Auditivo de Tronco Encefálico em casos de ossificação coclear e neuropatia auditiva

Vários estudos ${ }^{17,20-22}$ têm relatado os resultados obtidos em adultos e crianças com ossificação coclear. Todos eles obtiveram melhora na discriminação de fala e de outros sons.

Em um estudo ${ }^{21}$ os pacientes foram acompanhados durante seis anos. Foram ativados de $11 \mathrm{a}$ 15 eletrodos de um total de 22.

Em um caso ${ }^{22}$ foi relatado que uma criança de 12 anos obteve respostas tão satisfatórias que oito meses após a ativação dos eletrodos já utilizava o telefone.

Os benefícios constatados na comunicação após a implantação do implante auditivo de tronco encefálico foram: restabelecimento da percepção auditiva de sons ambientais e a estimulações com instrumentos sonoros, reconhecimento de palavras, reconhecimento de sentenças utilizando apoio audiovisual ${ }^{20}$, significativa melhora na discriminação da fala e dos sons em geral 17,21,22 e utilização do telefone ${ }^{22}$.

Resultados mais detalhados sobre os benefícios foram relatados por outro estudo ${ }^{20}$. Em adultos: $100 \%$ de reconhecimento de sentenças nos sujeitos que restabeleceram a percepção auditiva; acertos de $40 \%$ a $80 \%$ no reconhecimento de palavras de 6 a 8 meses pós ativação dos eletrodos; detecção de $80 \%$ dos instrumentos sonoros quatro meses após a ativação. A criança obteve $100 \%$ de detecção de sons instrumentais e percepção de palavras.

\section{DISCUSSÃO}

A utilização do implante auditivo de tronco encefálico proporciona o restabelecimento da sensação auditiva. Pacientes com perda auditiva profunda decorrente de patologias onde antes não havia chances com o uso de aparelhos auditivos ou implante coclear ${ }^{3}$ podem voltar a ouvir. Esta habilitação/reabilitação contribui para a melhoria da qualidade de vida de indivíduos privados da audição, diminuindo suas conseqüências ${ }^{1,2}$.

Perdas auditivas profundas determinadas por comprometimentos nas vias auditivas periféricas agora não são mais vistas como sem solução para a audição desde que a via auditiva central esteja preservada.

O implante auditivo de tronco encefálico realmente restabelece a audição em pacientes com perda auditiva total, proporcionando sensações auditivas ${ }^{12}$, níveis de detecção e discriminação dos sons similares às fornecidas pelo implante coclear ${ }^{13}$. A inteligibilidade da fala propiciada pelo implante costuma ser mais clara se associada à leitura labial ${ }^{3,14}$.

Nos estudos realizados, as habilidades comunicativas melhoraram para vários dos sujeitos implantados. Em adultos a estimulação permite reconhecimento de sentenças com e sem apoio visual ${ }^{3,17-21}$. Crianças passam a responder aos testes instrumentais, reconhecer vozes ${ }^{3} \mathrm{e}$ utilizar o telefone ${ }^{22 .}$

Como é possível concluir a partir de todos os estudos referidos, a implantação do implante auditivo de tronco encefálico na grande maioria das vezes é acompanhada de grande sucesso e devolve ao paciente a esperança de participar do mundo dos sons.

\section{CONCLUSÃO}

O implante auditivo de tronco encefálico é uma intervenção ambiciosa no campo da reabilitação auditiva, pois faz com que o estímulo sonoro seja captado diretamente pelos núcleos centrais do nervo auditivo.

O implante auditivo de tronco encefálico pode devolver a sensação auditiva em casos em que há lesão coclear e/ou na via auditiva até o tronco encefálico, o que anteriormente não era possível utilizando o implante coclear. 


\begin{abstract}
Purpose: to study important aspects about auditory brainstem implant, such as concept, purpose, methods of implantation, indication of the procedure and benefits. Methods: a compilation was carried out of the available works in LILACS, MEDLINE and SCIELO databases, using the descriptors: auditory brainstem implantation, rehabilitation of hearing impaired, sensorineural hearing loss, otologic surgical procedures. Results: the auditory brainstem implant is similar to cochlear Implant, except for the configuration of the electrode that is designed to be placed in the cochlear nucleus complex, at the brain stem. It was developed originally to restore the hearing in patients with absence of or injury in the cochlear nerve. Today it is indicated for patients who for anatomical or functional reasons can not receive electric stimulations by the inner ear, as in cases of malformation/absence of cochlear nerve, ossified cochleae, and auditory neuropathy. The effects showed are hearing recovery in patients with total hearing loss, levels of detection obtained discrimination of sounds similar to those obtained with cochlear Implant and access to environmental and speech sounds. Conclusions: the auditory brainstem implant provides hearing recovery in subjects that are not benefited by the use of hearing aids and cochlear Implant. Almost the totality of the implanted patients got some communication benefit.
\end{abstract}

KEYWORDS: Auditory Brain Stem Implantation; Rehabilitation of Hearing Impaired; Hearing Loss, Sensorineural; Otologic Surgical Procedures

\section{REFERÊNCIAS}

1. Almeida K. Avaliação dos resultados da intervenção. In: Almeida K, lório MCM. Próteses auditivas: fundamentos teóricos e aplicações clínicas. 2. ed. São Paulo: Lovise; 2003. p. 335-56.

2. Oliveira TMT, Casarin MT, Souza MA, Marquett SCE, Barros TN. Atitudes de médicos pediatras em relação à audição infantil. Pediatr Atual. 1998; 11(9):48-56.

3. Colletti V, Carner M, Miorelli V, Colletti L, Guida $M$, Fiorino F. Auditory brainstem implant in posttraumatic cochlear nerve avulsion. Audiol Neurootol. 2004; 9(4):247-55.

4. Bento RF, Sanchez TG, Brito Neto RV. Critérios de indicação de implante coclear. Arq Fund Otorrinolaringol. 1997; 1:66-7.

5. Shannon RV, Fayad J, Moore J, Lo WW, Otto $S$, Nelso RA. Auditory brainstem implant (ABI): post surgical issues and performance. Otolaryngol Head Neck Surg. 1993; 108:634-42.

6. Briggs RJ, Fagan P, Atlas M, Kaye AH, Sheehy $\mathrm{J}$, Hollow R, et al. Multichannel auditory brainstem implantation: the australian experience. J Laryngol Otol Suppl. 2000; (27):46-9.

7. Toh EH, Luxford WM. Cochlear and brainstem implantation. Otolaryngol Clin North Am. 2002; 35(2):325-42.

8. Brito Neto RV, Bento RF, Yasuda A, Ribas GC, Rodrigues Junior AJ. Referências anatômicas na cirurgia do implante auditivo de tronco cerebral. Rev Bras Otorrinolaringol. 2005; 71(3):282-6.
9. Fayad JN, Otto SR, Brackmann DE. Auditory brainstem implants: surgical aspects. Adv Otorhinolaryngol. 2006; 64:144-53.

10. Vautrin R, Mertens $P$, Streichenberger N, Ceruse $\mathrm{P}$, Truy E. Abord et repárage oto-neuro-chirugicaux des noyaux cochléaires: intérêt dans lïmplantation auditive du tronc cerebral. Rev Laryngol Otol Rhinol. 1998; 119:171-6.

11. Sollmann WP, Laszig R, Marangos N. Surgical experiences in 58 cases using the Nucleus 22 multichannel auditory brainstem implant. J Laryngol Otol Suppl. 2000; (27):23-6.

12. Brackmann DE, Hitselberger WE, Nelson RA, Moore J, Waring MD, Portillo F, et al. Auditory brainstem implant: I. Issues in surgical implantation. Otolaryngol Head Neck Surg. 1993; 108(6):624-33.

13. Neil LD, Jamie MR, James CM. Cochlear and auditory brainstem implants in the management of acoustic neuroma and bilateral acoustic neurofibromatosis. MJM. 1997; 3(2):115-20.

14. Otto SR, Brackmann DE, Hitselberger WE, Shannon RV, Kuchta J. Multichannel auditory brainstem implant: update on performance in 61 patients. $J$ Neurosurg. 2002; 96(6):1063-71.

15. Colletti V, Fiorino FG, Carner M, Sacchetto L, Miorelli V, Orsi A. Auditory brainstem implantation: the University of Verona experience. Otolaryngol Head Neck Surg. 2002; 127(1):84-96.

16. Grayeli AB, Bouccara D, Kalamarides M, AmbertDahan E, Coudert C, Cyna-Gorse F, et al. Auditory brainstem implant in bilateral and completely ossified cochleae. Otol Neurotol. 2003; 24(1):79-82. 
17. Colletti V, Carner M, Miorelli V, Guida M, Colletti L, Fiorino F. Auditory brainstem implant ( $A B I)$ : new frontiers in adults and children. Otolaryngol Head Neck Surg. 2005; 133(1):126-38.

18. Colleti V, Fiorino F, Sacchetto L, Miorelli V, Carner M. Hearing habilitation with auditory brainstem implantation in two children with cochlear nerve aplasia. Int J Pediatr Otorhinolaryngol. 2001; 60(2):99-111.

19. Colleti V, Carner M, Fiorino F, Sacchetto L, Miorelli V, Orsi $A$, et al. Hearing restoration with auditory brainstem implant in three children with cochlear nerve aplasia. Otol Neurotol. 2002; 23(5):682-93.
20. Colletti V, Carner M, Miorelli V, Guida M, Colletti L, Fiorino F. Cochiear implant failure: is an auditory brainstem implant theanswer? Acta Otolaryngol. 2004; 124(4):353-7.

21. Grayeli AB, Kalamarides M, Bouccara D, Ben Gamra L, Ambert-Dahan E, Sterkers O. Auditory brainstem implantation to rehabilitate profound hearing loss with totally ossified cochleae induced by pneumococcal meningitis.

Audiol Neurootol. 2007; 12(1):27-30.

22. Sanna M, Khrais T, Guida M, Falcioni M. Auditory brainstem implant in a child with severely ossified cochlea. Laryngoscope. 2006; 116(9):1700-3. 